\title{
Computing brains: learning algorithms and neurocomputation in the smart city
}

\section{Ben Williamson}

To cite this article: Ben Williamson (2017) Computing brains: learning algorithms and neurocomputation in the smart city, Information, Communication \& Society, 20:1, 81-99, DOI: 10.1080/1369118X.2016.1181194

To link to this article: http://dx.doi.org/10.1080/1369118X.2016.1181194
(c) 2016 The Author(s). Published by Informa UK Limited, trading as Taylor \& Francis Group

曲 Published online: 01 May 2016.

Submit your article to this journal $\widetilde{1}$

سل Article views: 1732

Q View related articles $\sqsubset$

View Crossmark data $־$

Citing articles: 1 View citing articles $\sqsubset$ 


\title{
Computing brains: learning algorithms and neurocomputation in the smart city
}

\author{
Ben Williamson \\ School of Social Sciences, University of Stirling, Stirling, UK
}

\begin{abstract}
This article examines IBM's 'Smarter Education' program, part of its wider 'Smarter Cities' agenda, focusing specifically on its learning analytics applications (based on machine learning algorithms) and cognitive computing developments for education (which take inspiration from neuroscience for the design of brain-like neural networks algorithms and neurocomputational devices). The article conceptualizes the relationship between learning algorithms, neuroscience, and the new learning spaces of the city by combining the notion of programmable 'code/space' with ideas about the 'social life of the brain' to suggest that new kinds of 'brain/code/spaces' are being developed where the environment itself is imagined to possess brain-like functions of learning and 'human qualities' of cognition performed by algorithmic processes. IBM's ambitions for education constitute a sociotechnical imaginary of a 'cognitive classroom' where the practices associated with data analytics and cognitive computing in the smart city are being translated into the neuropedagogic brain/code/spaces of the school, with significant consequences for how learners are to be addressed and acted upon. The IBM imaginary of Smarter Education is one significant instantiation of emerging smart cities that are to be governed by neurocomputational processes modelled on neuroscientific insights into the brain's plasticity for learning, and part of a 'neurofuture' in-the-making where nonconscious algorithmic 'computing brains' embedded in urban space are intended to interact with human cognition and brain functioning.
\end{abstract}

\section{ARTICLE HISTORY}

Received 28 August 2015

Accepted 8 April 2016

\section{KEYWORDS}

Algorithms; brain; cognitive computing; IBM; learning analytics; neurocomputation; neuroscience; smart cities

In recent years, urban environments have been reimagined as 'smart cities of the future' with the computational capacity to monitor, learn about, and adapt to the people that inhabit them (Batty et al., 2012). As geographers have detailed, the smart city is an urban environment governed by the capacities of coded devices and infrastructures (Kitchin, 2014a), a 'programmable environment' (Gabrys, 2014) structured and supported 'line by line, algorithm by algorithm, program by program,' 'by code using data as fuel' (Thrift, 2014, p. 10). To some degree, smart cities are even 'sentient' spaces that 'think

CONTACT Ben Williamson ben.williamson@stir.ac.uk $\mathrm{E}$ School of Social Sciences, University of Stirling, Stirling FK9 4LA, UK

(C) 2016 The Author(s). Published by Informa UK Limited, trading as Taylor \& Francis Group

This is an Open Access article distributed under the terms of the Creative Commons Attribution License (http://creativecommons.org/ licenses/by/4.0/), which permits unrestricted use, distribution, and reproduction in any medium, provided the original work is properly cited. 
of us' (Crang \& Graham, 2007, p. 792), with some form of reflexive awareness as learning environments. This article provides a case study of the 'Smarter Education' initiative of IBM's global 'Smarter Cities' program, focusing on its educational ambitions to create classrooms with in-built capacities for learning as an exemplar of its wider efforts to make cities into programmable learning spaces. Smarter Education itself is premised on technologies of learning analytics and cognitive computing. These are both highly algorithm-driven technologies: learning analytics depends on machine learning algorithms trained on data to analyse students' performance on tasks; cognitive computing relies on neural networks algorithms and associated neurotechnologies based on neuroscientific models of brain functioning, and has led to the creation by IBM of 'cognitive tutors' that can 'personalize' and 'optimize' learning according to each student's predicted progress. IBM's promise of the learning algorithms of educational data analytics and cognitive computing is to transform schools into 'smarter schools' - brainy spaces where the environment itself has been encoded with capacities for learning - which might act as templates for increasingly 'cognitive cities' that are 'configured for advanced mental processing' (Dunn, Cureton, \& Pollastri, 2014).

The learning algorithms of learning analytics and cognitive computing applications imagined by IBM contain particular models of learning processes that are themselves becoming active much more widely in how the smart city as a learning environment is enacted. The central contribution of the article is to identify how IBM is promoting processes of algorithmic learning that are integral to its vision of smarter cities. Its glossy imaginary of Smarter Education acts as a seemingly desirable model not just for the future of schools in software-enabled urban environments, but as a diagram for future cities that are to be treated as learning environments and enacted by increasingly cognitive forms of computing technology. Smarter Education is one significant instantiation of emerging smart cities that are to be governed by neurocomputational processes modelled (in part) on the brain's pliability for learning - but with the capacity to compute beyond human perceptibility and consciousness - wherein brain-based devices embedded in urban space are imagined to have the capacity to optimize human neural morphology and cognitive functioning. A reimagining of the human subject is instantiated by such developments. As Rose (2016) asks, why do some dream that new neurotechnologies will make it possible to 'read' the brain, what practical applications might such technologies lead to, and what mutation in our understandings of the human might result from their development? As an exemplar 'neurofuture-in-the-making' (Williams, Katz, \& Martin, 2011, p. 143), IBM's own agenda is based on a dream of modelling the brain, is leading to the design of new computational applications (exemplified in its education programs and its aspirations for more cognitive smart cities), and is contributing to a reimagining of the human subject - one understood algorithmically in terms of computable cognition and programmable brain functions.

\section{IBM imaginaries}

A number of organizations associated with smart cities have begun to produce materials envisaging education as a smart social institution situated in digitally mediated urban infrastructures. Like the city, the school is treated as a 'code/space' (Kitchin \& Dodge, 2011) in these imaginings, where code and algorithms play an integral part in the functioning of the 
built environment itself. For Kitchin and Dodge (2011) the term 'code/space' articulates how the functioning of spaces is translated into coded processes that then act recursively to alter them. Elsewhere, Kitchin (2014a) refers to the 'programmable city' as a prototypical code/space, where urban functions are delegated to software systems which then transform how they perform. However, the educational dimensions of smart cities remain empirically neglected and critically under-conceptualized. This is significant since the smart city visions of major software vendors such as IBM and Microsoft posit a direct relationship between education and the future city - not just in educating 'smart citizens' to participate in it (Williamson, 2015), but insofar as they treat programmable urban spaces as learning environments that utilize the power of machine learning and cognitive computing algorithms to learn about the people and things that inhabit them.

As a way of opening up this gap into a research agenda around education in smart cities, I analyse the 'sociotechnical imaginary' of IBM's Smarter Education program. Sociotechnical imaginaries are collectively held, institutionally stabilized, and publicly performed visions of desirable futures that are animated by imagined forms of life and made attainable through the design of technological projects (Jasanoff, 2015). The code/ spaces of smart cities are in this sense sociotechnical imaginaries materialized and operationalized through specific technological innovations and practices. In particular, I focus on IBM as a key organizational actor that has emphasized learning analytics and cognitive computing in its global Smarter Education program. Smarter Education articulates an imagined future for educational spaces and practices being operationalized by IBM in specific technological projects: it acts as a model for the future of schooling that IBM assumes is both attainable and should be attained through the application of technical products, and that also illustrates how algorithmic forms of learning are imagined by IBM as integral to the functioning of the smart cities to which it is seeking to sell its technical solutions.

Methodologically, researching IBM's imaginary has involved searching its promotional and scientific literatures, websites, infographics, press releases, and news features, and tracing the connective tissues between IBM's scientific claims and knowledges, its human actors, its technical applications, and its production of visions of a future in which its applications are to be operationalized. While cautious that much of this material is largely promotional and serves IBM's marketing aspirations as a global smart cities software solutions vendor, I emphasize how its materials 'frame and represent alternative futures' and 'naturalize ways of thinking about possible worlds' and associated forms of social life and behaviour (Jasanoff, 2015, p. 35). In particular, I explore what models of learning and brain functioning underpin IBM's production of machine learning and cognitive computing applications, critically considering their possible consequences for the shaping of desired behaviours and practices in an imagined neurofuture of education. These are algorithmic processes being mobilized in the present that possess both a past life in the practices of IBM's R\&D labs and a future life in the IBM imaginary of Smarter Education. While empirical data on the smarter classrooms imagined by IBM are not currently available, by analysing the sociotechnical imaginary of IBM's Smarter Education initiative I examine how learning algorithms might participate in the smart city itself by advancing the idea of 'brain/code/space.' Brain/code/space articulates how environments are becoming increasingly programmable, but also how such spaces are becoming dependent upon encoded models of human cognitive functioning to become 
adaptive learning environments, and represents the material and spatial instantiation of imagined computational neurofutures-in-the-making.

\section{Modelling algorithms}

Learning analytics and cognitive computing are both fundamentally algorithm-driven. A growing body of research has begun to engage with algorithms both as social products designed by technical experts in specific social settings - and as socially productive systems that interact with diverse practices. As products, Kitchin (2014b) characterizes algorithms variously as 'black boxes' that are hidden inside intellectual property and proprietorial code; as 'heterogeneous systems' in which hundreds of algorithms are woven together; as 'emergent' systems that are constantly being refined, reworked, and tweaked; and as complex, unpredictable, and fragile systems that are sometimes miscoded, buggy, and 'out of control.' Beyond their properties as products, these vastly complex algorithmic systems can then 'do things,' and exert material effects 'on themselves, on machines and on humans' (Goffey, 2008). As a consequence, algorithms are becoming an integrated part of everyday social processes that can reinforce, maintain, or even reshape visions of the social world, knowledge, and encounters with information (Beer, 2013).

It is important to distinguish such claims from simplistic technological determinism, and to acknowledge algorithms as products of social practices. Gillespie (2014, n.p.) argues that 'sociological analysis must not conceive of algorithms as abstract, technical achievements, but unpack the warm human and institutional choices that lie behind these cold mechanisms.' In particular he highlights the importance of examining how complex human and social activities - and the values and assumptions held about them - are operationalized by being translated into a functional interaction of models, goals, data, variables, indicators, and outcomes. The algorithm itself, in this sense, may not be as important an object of inquiry as the underlying 'models' - including models of human action - on which algorithms are intended to operate within 'the social world of the algorithmic system' (Neyland, 2015, p. 128).

In the below examples, the emphasis is on those models of human learning and cognition that are built into the learning analytics systems and cognitive classroom spaces being developed by IBM. While it is beyond the scope of this article to penetrate the technical complexity of IBM's algorithmic systems, it is possible to analyse the available documentation emerging from IBM to discern what kinds of assumptions, models, and desired outcomes its algorithms are intended to operationalize. IBM's imaginary of Smarter Education is a documentary construction of a desired future world 'out there' that IBM is seeking to build 'in here, in the algorithmic machine' (Neyland, 2015, p. 129).

\section{Learning analytics}

Learning analytics software is designed to enable students to be tracked through their digital data traces in real time and to provide automated predictions of future progress (Siemens, 2013). Fundamentally interdisciplinary, the emerging field of learning analytics consists of expertise in statistics, computer science, information science, machine learning, psychology, and neuroscience (Piety, Hickey, \& Bishop, 2014). Within the field itself, learning analytics is often considered in terms of both its techniques and applications 
(Siemens, 2013). Techniques involve the specific algorithms and models for conducting analytics; applications involve the ways in which insights generated from analytics are then codified into software products to improve teaching and learning. As Siemens (2013, p. 1386) details, 'an algorithm that provides recommendations of additional course content for learners can be classified as a technique. A technique, such as prediction of learner risk for dropout, can then lead to an application, such as personalization of learning content.'

A key organizational developer of learning analytics techniques and applications is IBM, which houses its learning analytics R\&D within its Smarter Education program, itself a subtheme of its global Smarter Cities agenda. Smarter Education is based on a series of assumptions about the real-time availability of educational data about what students learn and how they progress, and about the beneficial uses of data analytics for institutional and systemic improvement (IBM, 2015a). According to its paper on 'the future of learning':

Analytics translates volumes of data into insights for policy makers, administrators and educators alike so they can identify which academic practices and programs work best and where investments should be directed. By turning masses of data into useful intelligence, educational institutions can create smarter schools for now and for the future. (IBM, 2014)

In detailing its imaginary of 'smarter schools,' the report particularly emphasizes the use of 'academic analytics' to enable institutions to analyse data for insights into their effectiveness, and 'learning analytics' to facilitate the interpretation of students' actions. These analytics include both 'predictive tools' - which model probable future progress on data from past activities - and prescriptive analytics - which automate appropriate pedagogic responses: 'these two dimensions of smarter analytics enable educational leaders to detect patterns that exist in masses of data, project potential outcomes and make intelligent decisions based on those projections' (IBM, 2014, n.p.). To this end, IBM has established its own high school chain in the US, P-TECH, which exemplifies how schools could generate real-time data on student activities, but also make future-tense predictions of their likely outcomes and prescribe pedagogic interventions. The ambition of P-TECH is 'to build for schools what its operations center is for cities: a single system for collecting, aggregating and analyzing data from students and teachers alike, then writing algorithms to prescribe how to cope' (Linday, 2013, n.p.). As an operationalization of the Smarter Education imaginary, P-TECH makes every aspect of institutional and individual performance into a real-time and future-focused process of data collection, analysis, and feedback.

Given the focus in this paper on the modelling that must take place for algorithms to function, it is important to note that learning analytics is fundamentally underpinned by techniques of user modelling. For example, Siemens (2013, p. 1386) explains how 'new data-based discoveries are made and insight is gained into learner behavior ... through models and algorithms.' Learner modelling, cognitive modelling, behaviour modelling, probability modelling, and 'knowledge domain modelling' (the mapping of the knowledge structure of a discipline) are crucial elements in any learning analytics platform. Once these models are combined, they can be used to produce predictive models of learner progressions. Here machine learning algorithms and predictive analytics are significant. 'Machine learning' consists of software systems that utilize adaptive algorithms, 
techniques of 'deep learning,' and statistical models to analyse users' data and anticipate or even predict their future actions by 'transforming data on events, actions, behaviours, beliefs and desires' into probabilistic predictions of the future that then can be used to decide on action to be taken in the present (Mackenzie, 2013, p. 399). The practical 'production of prediction,' as Mackenzie (2015a, p. 436) terms it, depends on a range of mathematical, statistical, logistic, and calculative practices that are rooted in particular predictive styles and machine learning settings, and situated in the province of experts such as engineers, mathematicians, and statisticians working in university and industry research. Predictive modelling is thus highly contingent on the situatedness of its production in fields of technical expertise and experimentation.

It is important to reiterate that the object of inquiry here is the interaction of the algorithm with the underlying model that has been constructed from the data. As Gillespie $(2014$, n.p.) notes, 'the "algorithm" comes after the generation of a "model," i.e. the formalization of the problem and the goal in computational terms.' Moreover, in order for the algorithm to function - particularly the case with machine learning algorithms - it must first be 'trained' with existing data 'so that it may "learn"' (Gillespie, 2014), and need to be constantly re-trained in an iterative process of monitoring, adjusting, revising, and optimizing as the accuracy and generalizability of the predictive models it generates are themselves checked and analysed (Mackenzie, 2015a). The models and the training data are always constructed and operationalized according to the values and assumptions of their designers. Fundamentally, learning analytics such as those being developed and deployed by IBM depend on the construction of models of learner actions, and learning processes, which can then be subjected to algorithmic processes that have themselves been designed to learn. These models are the product of complex sociotechnical practices and are embedded in the methodological commitments, assumptions, values, and styles of thinking of their designers, such as those associated with Smarter Education at IBM.

Although the specific internal practices of IBM are beyond the empirical scope of this study, it is possible to see from its documentary resources how a particular set of assumptions about the use of learning analytics and machine learning algorithms is circulating within the institutional context of the company. There is, in other words, an institutional social life to the algorithmic data practices that IBM is developing as part of Smarter Education. From the documentary traces that constitute its imaginary of smarter schools, it is possible to discern that IBM is seeking to model different aspects of education, including practices of learner modelling and behaviour modelling, as a means towards generating predictions of future actions and outcomes. Further empirical investigation of these practices would seek to examine the institutionalized processes involved in training and re-training the machine learning algorithms that will interact with those models to generate insights into the behaviour of the learner and to make the learning process known and thus amenable to intervention. One significant modelling technique being developed by IBM to this end is cognitive modelling of the neural structure of the 'learning brain.'

\section{Cognitive classrooms}

An emerging development in IBM's learning analytics and associated machine learning techniques is 'cognitive-based learning systems' based on neuroscientific methodological innovations, technical developments in brain-inspired computing, and neural networks 
algorithms. IBM's promotion of cognitive learning systems within education is part of a proliferating discourse of educational neuroscience, or 'the dispersal of neurobiological language, imagery, symbolism and rhetoric within formal and informal learning environments' (Busso \& Pollack, 2015, p. 169). The discourse of contemporary education is increasingly infused with references to 'neuroscience in education,' 'neuroeducation,' and 'neuropedagogies.' These terms reflect how neuroscientific understandings of the learning process have been used to inform the design and application of better pedagogies, though many neuroeducational approaches treat the functional architecture of the brain in explicitly determinist terms, and even 'reduce learning to an algorithmic or computational process' (Pykett, 2015, p. 97). In applying its expertise in cognitive computing to education, IBM is taking a particular algorithmic model of brain functioning as the basis for imagining new neurocomputational systems that might intervene in young people's own learning processes - processes taking place in a 'learning brain' that itself has been conceptualized algorithmically through IBM's own laboratory studies.

Cognitive modelling of the kind promoted by IBM has long been an aspiration of learning analytics developers. Cognitive modelling is concerned with developing systems that possess a 'computational model capable of solving the problems that are given to students in the ways students are expected to solve the problems,' and thus since 'cognitive processes can be modeled, software (tutors) can be developed to support learners in the learning process' (Siemens, 2013, pp. 1383-1384). With the current development by IBM of cognitive-based learning technologies built on the idea that the architectures and functions of the brain can now partly be modelled computationally, cognitive modelling is seen within the learning analytics field as more attainable. It is seen as possible to create technologies that function more like human brains than programmed software; technologies that can then be embedded into schools as a cerebral augmentation to the cognitive capacities of the learner. The promise here is of technologies that can learn from the user, through processing data collected during their digital learning activities, and then adapt and respond to that user's individual needs, preferences, and dispositions in ways that are more 'natural' than hard-programmed computing systems. IBM's cognitive systems, its advocates claim, 'learn at scale, reason with purpose and interact with humans naturally,' thanks to their 'human qualities, such as self-directed goals, common sense and ethical values' (Kelly, 2015, p. 5).

Over the last decade, IBM has positioned itself as a dominant research centre in cognitive computing, with huge teams of engineers and computer scientists working on both basic and applied research in this area. The development of cognitive computing with 'human qualities' at IBM - again linked to its Smarter Cities agenda - closely mirrors current scientific R\&D around 'neural networks' algorithms and 'neuromorphic' hardware in the analysis of big data. The emerging discourse of cognitive computing is replete with references to the brain as a 'big data processor,' 'brain-like computations,' 'algorithms that learn,' 'neural network learning algorithms,' 'brain-inspired algorithms,' and 'deep learning algorithms.' Building on such developments, IBM has positioned cognitive computers as 'decision support systems' that 'can process natural language and unstructured data and learn by experience, much as humans do,' in contrast to expert systems that are hard coded by programmers (IBM Research, 2015, n.p.). It has even produced its own visionary book, Smart machines: IBM's Watson and the era of cognitive computing (Kelly \& Hamm, 2014). Perhaps the most well-known IBM cognitive computing 
development is Watson, a massively advanced cognitive supercomputer promoted by IBM for its capacity to process and learn from natural language and other unstructured data:

Watson is a cognitive technology that processes information more like a human than a computer - by understanding natural language, generating hypotheses based on evidence, and learning as it goes. And learn it does. Watson 'gets smarter' in three ways: by being taught by its users, by learning from prior interactions, and by being presented with new information. (IBM, 2015b, n.p.)

Watson has become the subject of almost feverish R\&D both within IBM and among the vast network of partners and subsidiaries in the 'Watson ecosystem' of application developers, content providers, and 'talent partners.' Watson has already been applied in healthcare, higher education, the culinary sector, banking, and business, as well as in 'citizen services' in cities (Terdiman, 2015). IBM's wider R\&D network in cognitive computing is based on many years of neuroscientific research at its 'Brain Lab,' much of it supported by the US Defense Advanced Research Projects Agency (DARPA), as part of its Systems of Neuromorphic Adaptive Plastic Scalable Electronics (SyNAPSE) program to build 'artificial brains,' with hundreds of millions of US dollars funding (Artificial Brains, 2013).

Much of IBM's cognitive computing R\&D is based on the neo-cortical principles of the brain as a synaptic memory system determined by computable neuronal patterns, rather than human-engineered architectures. Its Brain Lab has provided the neuroscientific insight for these developments. While IBM itself is not seeking to build an artificial brain but 'a computer inspired by the brain,' it claims that 'cognitive computing aims to emulate the human brain's abilities for perception, action and cognition,' and has dedicated extensive R\&D to the production of 'neurosynaptic chips' that can 'emulate the neurons and synapses in the human brain' (IBM Research, 2014, n.p.). In 2014 IBM engineers published a major article in Science that was featured in its leader comment and front cover (Merolla et al., 2014; Service, 2014). In a series of related articles in both specialist and non-specialist publications, IBM engineers claimed to have created a 'one million neuron brain-inspired processor,' a 'brain chip' that is 'capable of 46 billion synaptic operations per second, per watt-literally a synaptic supercomputer in your palm' (Modha, 2014, n.p.). These neurosynaptic chips can also be tiled together into 'scalable neuromorphic systems' of several millions of neurons and billions of synapses, referred to in promotional IBM literature as 'computing brains,' 'systems that can perceive, think and act,' or even a 'brain-in-a-box' at a 'roughly human scale' (Modha, 2013, n.p.).

The elision of computation and the brain by IBM is both a marketing strategy and an engineering innovation. As marketing, it positions IBM as a solutions-provider for complex data analysis problems. As an engineering innovation, it proposes a new model for computation that, according to the Science article, displaces previous 'programmable' approaches to algorithm design and machine learning. Whereas conventional machine learning algorithms depend on being programmed and trained with example data (sometimes termed 'supervised learning'), cognitive computing systems such as IBM's brain chip are designed with the capacity to process and learn from natural language, interactions with users, and other unstructured data ('unsupervised learning') in ways that emulate the neural networks of the human brain. The development path of IBM's 'brain chip' and 'computing brains,' recorded in a series of published scientific articles, has proceeded from neuroscience and neuroanatomy to supercomputing, to a 
new computer architecture, to a new programming language, to algorithms, and applications, all underpinned by its understanding of the human brain's synaptic plasticity developed within IBM's own Brain Lab. Plasticity is the understanding that the brain's neuronal architecture is itself pliable, flexible, and constantly adapting to environmental input, and is the basis for much neural networks R\&D. IBM's cognitive computers are, therefore:

designed to learn dynamically through experiences, find correlations, create hypotheses and remember - and learn from - the outcomes, emulating the human brain's synaptic and structural plasticity (or the brain's ability to re-wire itself over time as it learns and responds to experiences and interactions with its environment). (IBM Research, 2011, n.p.)

IBM's engineers are modelling the neural plasticity of the learning brain in silicon.

To apply its cognitive computing applications in education, IBM has developed a specific 'Cognitive Computing for Education Transformation' program, led by its own 'Program Director and Master Inventor,' though care needs to be taken to differentiate the cutting edge of IBM's R\&D from the imaginaries of application that have quickly surrounded it. IBM's cognitively 'smarter classroom' initiative is just one such imaginary application of cognitive computing, part of a series of 'visionary' scenarios for the future that also include healthcare, retail, security, and smart cities that are all imagined as being responsive, real-time, predictive, and highly personalized. Like the IBM smart cities into which it is integrated in these scenarios, IBM's imaginary of a 'smarter classroom' is intended to use cognitive computing applications to analyse massive quantities of student data in real time and then wrap personalized learning experiences around each one:

IBM envisions educational institutions adopting cloud-based cognitive systems to collect and analyse all of this data over a long period of time - creating longitudinal student records that would give teachers the information they need to provide personalized learning experiences for their students. (IBM Research, 2013a, n.p.)

In its imaginary of the classroom in five years, IBM grandly claims that the IBM 'smarter classroom' is a 'classroom that will learn you' through 'cognitive-based learning systems.' As the IBM promotional website for the 'classroom that will learn you' claims:

The rapid digitization of educational institutions will allow unprecedented instrumentation of the learning process. Cognitive computing, or learning technologies, will help us calculate everything we can about how each student learns and thrives, then create flexibility in the system to continually adapt and fine-tune what we deliver to that student and how this supports teachers and employers. (IBM, 2013, n.p.)

These claims are reinforced and reiterated in a variety of IBM think pieces, glossy interactive multimedia presentations, and infographics available on the company website. The cognitive classroom promises personalization of the learning experience, real-time feedback on learner performance, adaptive learning software that can learn from and adapt to the learner, and intelligent software tutors that can automate remedial intervention or even prescribe appropriate curricular content.

IBM's Cognitive Computing for Education Transformation program director has presented these as intelligent, interactive systems that combine neuroscientific insights into cognitive learning processes with neurotechnologies that: 
learn and interact with humans in more natural ways. At the same time, advances in neuroscience, driven in part by progress in using supercomputers to model aspects of the brain ... promise to bring us closer to a deeper understanding of some cognitive processes such as learning. At the intersection of cognitive neuroscience and cognitive computing lies an extraordinary opportunity ... to refine cognitive theories of learning as well as derive new principles that should guide how learning content should be structured when using cognitive computing based technologies. (Nitta, 2014, n.p.)

The prototype innovations so far developed by this program include automated 'cognitive learning content,' 'cognitive tutors,' and 'cognitive assistants for learning'; these may be integrated into 'personalized adaptive learning systems,' all 'designed with a deep understanding of underlying cognitive neuroscience as well as cognitive theories of learning' (Nitta, 2014, n.p.), to provide intelligent computational augmentation to the learner's cognitive process. As the IBM Global Manager of Education Solutions for Smarter Cities phrases it, the 'cognitive tutor' application is intended

to supplement face-to-face teaching and ultimately replace it entirely for subjects and areas where a cognitive agent will, quite simply, do a better job of understanding the learner's needs and provide constant, patient, endless support and tuition personalized for the user. (Eassom, 2015, n.p.)

IBM has also developed an application based on Watson, called Codename: Watson Teacher Advisor, which is designed to observe, interpret, and evaluate information to make informed decisions that should provide guidance and mentorship to help teachers improve their teaching.

The imaginary of the cognitive tutor and the teacher advisor clearly resonates with a longer genealogy of thinking about the automation of teaching (Bayne, 2015), but more uniquely superimposes an emergent computational theory of the brain on to the spaces in which education takes place. The promise of the classroom that can learn you is of a smart environment with its own cognitive faculties, designed according to neuroscientific claims about the brain as a sophisticated algorithmic system that is defined by its capacity as a synaptic memory system that 'spikes' as it receives inputs. It treats the cognitive classroom and the human subjects that inhabit it in increasingly analogous terms. The promise of cognitive computing for IBM is not just of more 'natural systems' with 'human qualities,' but a fundamental reimagining of the 'next generation of human cognition, in which we think and reason in new and powerful ways,' as claimed in a recent IBM white paper entitled 'Computing, cognition and the future of knowing':

It's true that cognitive systems are machines that are inspired by the human brain. But it's also true that these machines will inspire the human brain, increase our capacity for reason and rewire the ways in which we learn. (Kelly, 2015)

The key point here is that IBM's imaginary of smart learning environments has distinctive implications for thinking about smart cities as brain-inspired computational spaces in which the human cognitive capacities of the learning brain might be enhanced. The neurocomputationally cognitive classroom that can learn is part of IBM's emerging vision of an 'ecosystem of cognitive environments inhabited by a society of specialized software agents called cogs': 
Cogs work in a mutually beneficial partnership with humans to enable better complex datadriven decision-making. We call these partnerships Symbiotic Cognitive Systems. Cognition does not occur solely (or even mostly) within an individual human mind, but rather is distributed across people, artifacts and environments. ... Cogs are designed to follow and interact with humans and other cogs across a variety of everyday environments. They engage individually or collectively with humans ... [and] learn and leverage sophisticated models of human characteristics, preferences and biases so they can communicate naturally. (IBM Research, 2013b, n.p.)

IBM's imaginary of the cognitive ecosystem, or a 'symbiotic cognitive system,' is underpinned by an 'infrastructure inhabited by the society of cogs and the devices that let them behave as one shared integrated resource, enabling "human-computer collaboration at the speed of thought"' (IBM Research, 2013b, n.p.). Its cognitive environments include cognitive homes, cognitive offices, cognitive cafes, and increasingly cognitive cities within which each of these environments are networked together. The cognitive classroom that can learn you is one example of such a 'symbiotic cognitive system,' in which cognitive software agents are enabled to interact with users, and to participate in a distribution of cognition across the neurobiology of the human brain and the neurosynaptic devices of cognitive computing. As with the smart city itself, the cognitive environment of the classroom constitutes a code/space where coded devices and infrastructures partially determine individual actions and social activities. Crucially, the cognitive code/spaces imagined by IBM put brain-inspired algorithms directly into interaction with human cognition, mobilizing machine models of neuronal network processes to extend and optimize the capacities of the human mind itself.

Mackenzie (2015b, n.p.) has argued that advances in cognitive computing in places like IBM are based around 'the ideal of something like pattern recognition or indeed conscious awareness' and 'abound in references to cognition, meaning, perception, sense data, hearing, speaking, seeing, remembering, deciding, and surprisingly, imagining and fantasy.' As such, Mackenzie (2015b, n.p.) claims, their 'modelling practices are no longer the statistical rendering of number in the hands of government, science or commerce' but 'as challenges set for an often almost Cyclopean cognition to reorganise and optimise.' Mackenzie terms such technologies 'cognitive infrastructures.' IBM's imaginary of the cognitive classroom can be conceived, then, as a cognitive infrastructure in which cognitive tutors, cognitive learning content, and cognitive assistants are powered by neurocomputational learning algorithms that can be applied to optimize learners' cognition. In such fabricated spaces, the brain functioning of human subjects themselves is rendered reductively as algorithmic or computational processes, which are therefore amenable to being optimized by the application of cognitive computing algorithms. The smarter school is imagined as a brainy space which is located in the cognitive infrastructure of a neurocomputationally smart city that is itself animated by neuroscientific models of the plastic brain but networked together into an ecosystem of distributed intelligences that can out-compute the capacities of human perception, consciousness, and cognition.

\section{Modelling, morphing, and modifying the mind}

The application of 'brain-inspired thinking' in IBM's neuropedagogic plans for the cognitive classroom reflects the increasingly prominent application and popularization of 
'neuro knowledges' (Pykett, 2013) - those disciplines of the brain sciences with their own styles of thinking, explanations, modes of expertise, and application. However, the different branches of the neuro knowledges assume different working models of the brain, mobilize different methods to measure it, and build different theories and empirical accounts of its functioning, all shaped by specific social, political, cultural, and economic contexts (methodological and technical innovations, political and industrial support, etc.) (Rose \& Abi-Rached, 2013). Additionally, much contemporary neuroscience attributes capacities to the brain from experimental findings in artificial laboratory contexts, as 'brain facts' abstracted from embodied life and culture (Rose, 2016; Williams et al., 2011). Neuroeducation, in particular, tends to treat brain processes as if they are computational or algorithmic processes, and is part of an emerging field of policy experimentation that treats reductive forms of neuroscientific evidence about the neurobiological processes of young people as the target for strategic intervention (Pykett, 2015). Through the 'biopolitics of the brain sciences,' the 'cerebral knowledges' of neuroscience are coming to play a significant role in contemporary techniques of governance, whereby 'experts of the brain, rather than of "psy" or society,' are understood to be addressing societal challenges, and 'governing the conduct of human beings [has] come to require, presuppose and utilize a knowledge of the human brain' (Rose \& Abi-Rached, 2014, pp. 3-5). As a result, neuroscientific developments have become entwined with strategies designed to govern human conduct by drawing upon the growing availability of empirical knowledge of the brain.

In recent critical science and technology studies, neuroscience has been conceptualized in terms of its 'numerous interpretations, translations, and mediations' rather than an assumed 'neuro-realism' (Williams et al., 2011, p. 139). The neuro knowledges emerging from IBM's Brain Lab and applied in its cognitive computing applications therefore need to be seen as socially situated practices providing a particular neurocomputational model and knowledge of brain processes. Its activities position it as a solutions-provider at a time when governments are seeking to intervene in human lives through the brain itself, not least in education and in response to problems of urban living (Fitzgerald \& Rose, 2015). If the social power of algorithms now plays a part in organizing everyday life (Beer, 2013), then the algorithmic power of brain-based cognitive computing in particular poses significant issues for the ways individuals and collectives are targeted for intervention through human-computer interaction with learning algorithms. The IBM imaginary of cognitive computing in education is part, therefore, of a much wider emerging debate about the influence of neuroscientific thinking in addressing societal challenges and shaping human subjectivity, though care is required to differentiate simplistic claims about brain manipulation from the strategic uses of neuroscientific concepts by political and commercial actors, including IBM, that are increasingly mobilizing neuro expertise in the computational modelling of brain processes as the basis for intervening in the cognitive lives of human subjects.

As noted earlier, one of the key concepts of the brain sciences is neuroplasticity. The understanding of the brain's malleability has become a dominant neuroscientific claim, not just at IBM's Brain Lab. Methodological inquiries have ascertained that the brain is open to environmental input, with the environment shaping the neural architecture and functional organization of the brain through the formation, strengthening and trimming of synaptic connections. As a result of recent discoveries around neural plasticity, there is 
emerging consensus that neurobiological mechanisms exist through which 'environments get encoded in brains' and aspects of social life are incorporated into neurobiological structures (Fitzgerald \& Rose, 2015). The result of this emerging 'imaginary of plasticity' (Rose \& Abi-Rached, 2013) is that new techniques are now being devised to recognize and manage the processes involved in shaping and reshaping the brain which promote the idea that the brain is flexible, mouldable, able to be trained, re-wired, improved, and ultimately optimized. The imaginary of plasticity instantiates the social within the neurobiological, with new understandings of 'the social life of the brain' being used to animate policies and practices in healthcare, education, and other social domains (Pickersgill, 2013, p. 322).

IBM's cognitive classroom, as a cognitive environment inhabited by cognitive tutors, is illustrative of the imaginary of plasticity being materialized in pedagogic form. In this imagined space, cognitive systems that have been built to emulate the plasticity and neural networks of the brain are to be put to pedagogic work as cognitive tutors that might 're-wire' the neural circuits underpinning human learning itself. The expert basis for such systems emanates from IBM's own Brain Lab. Its neuroscientific and computational $\mathrm{R} \& \mathrm{D}$ practices have helped identify the neural networking of the social life of the brain itself, and developed ways of modelling such processes computationally in ways that have allowed its engineers to build new neurocomputational applications. Its neuro knowledges have been translated into neural network algorithms and neurosynaptic devices such as the brain chip that it seeks to apply in a social ecosystem of cognitive devices to extend the networks of the mind.

While IBM's elision of the brain and computation may appear dubious, it reflects recent sociological debate about the social life of the brain, as 'the webs of human social and cultural life that we had come to understand as our particular object of knowledge seem more and more open to being figured neuroscientifically' (Fitzgerald \& Callard, 2015, p. 4). Media researchers, too, have begun to examine how ideas about the social life of the brain, and its collapsing of taken-for-granted distinctions regarding 'biology' and 'society,' might imply a shift in understanding of human subjectivity. For example, Hayles (2013, p. 10) argues that recent discoveries around neural plasticity support the idea that humans develop through 'epigenetic changes - changes initiated and transmitted through the environment rather than through the genetic code', and therefore that humans and technologies can be understood to 'coevolve' together 'technogenetically':

As digital media ... embedded in the environment, become more pervasive, they push us in the direction of faster communication, more intense and varied information streams, more integration of humans and intelligent machines, and more interactions of language with code. These environmental changes have significant neurological consequences. (Hayles, 2013, p. 11)

Elsewhere, Hayles (2014, p. 202) refers to 'nonconscious cognitive systems' that increasingly permeate information and communication networks and devices, so that cognition in some instances may be located in a technical system rather than in the mental world of an individual participant, 'an important change from a model of cognition centered in the self.' This non-anthropocentric view of 'cognition everywhere' accepts that nonconsciously cognitive computing devices can employ learning processes that are modelled like those of embodied biological organisms, using their experiences to learn, achieve skills and interact with people. When nonconscious cognitive devices penetrate into 
human systems, they can then potentially change the dynamics of human behaviours through changing brain morphology and functioning technogenetically. Models of neural plasticity emerging from neuroscience have thus provided the imaginary necessary for the development of the nonconscious learning algorithms of cognitive computing, which are then used to activate cognitive environments such as IBM's cognitive classroom; a space in which learners are targeted for cognitive enhancement and neuro-optimization through interacting with other nonconscious cognitive agents and environments.

In IBM's imaginaries of Smarter Education and Cognitive Computing for Education Transformation, the brain is to be translated into computational code to enact nonconscious cognitive computing devices; and it is then to be applied in the code/space of the cognitive classroom to alter the functioning of the brain. This is symmetrical with Kitchin and Dodge's (2011) notion of how urban functions can be delegated to software code and algorithms to produce code/spaces that then fundamentally alter the functioning and experience of the city itself. In IBM's imaginary of the cognitive classroom - like that of the cognitive smart city - the human subject is approached as a plastic brain, but a plastic brain that is understood in terms of algorithmic and computational processes. This is a very specific form of neuro knowledge, or rather a neurocomputational knowledge, and it allows IBM to develop its ideas about symbiotic cognitive systems in which algorithmic forms of nonconscious cognition and learning as well as human cognition and learning can be optimized.

One way of conceptualizing the hybridity of programmable spaces with the neuroscientific figuring of the social life of the brain is the notion of a neurocomputational brain/ code/space.' This term registers how the learning algorithms of data analytics and cognitive computing are weaving constitutively into the functioning and experience of smart cities, including but not limited to the cognitive classrooms of IBM's imagined smarter school. The brain/code/spaces of IBM's smart cognitive classrooms are built around models of the brain that are encoded in the functioning of learning algorithms, inserted into the pedagogic space of the classroom, and that, located there, might act technogenetically to alter brain morphology and enhance cognitive functioning according to expert knowledges about the algorithmic nature of brain processes. IBM's imaginary of the brain/code/spaces of such cognitive learning environments is one instantiation of a new kind of urban cognitive infrastructure in which neuroscientific claims about brain plasticity are built into the learning algorithms that constitute the functioning and experience of the environment itself. There is some resemblance here with recent accounts of biopolitics in which 'the body is increasingly seen not as an organic substratum but as molecular software that can be read and rewritten' (Lemke, 2011, p. 93). The notion of brain/ code/space articulates a novel neurocomputational biopolitics in which brain functions are transcoded into data, and then codified into nonconscious cognitive learning algorithms and applications that are designed to augment human cognition. In sum, IBM's claims about the cognitive classroom represent a nexus of neuro knowledges and imagined neurofutures with technical expertise in learning algorithms, neural networks, cognitive computing, and neurosynaptic modelling. These approaches assume that it is now possible to understand and model the learning brain, one that is computationally understood and mapped in terms of the plasticity of its synaptic connections and neural pathways, and transcode it into learning algorithms that can be embedded in the environment. An imagined form of life based on computational models of the plastic, programmable, and 
optimizable brain animates the technological projects that are transforming IBM's imaginary of Smarter Education into a neurofuture-in-the-making.

\section{Conclusion}

This article has offered an initial exploration of the neurocomputational hybridity of technological code/spaces with new conceptions of the plasticity of the 'social life of the brain.' Through an examination of the IBM imaginary of Smarter Education as an exemplar of its aspiration for Smarter Cities, it suggests that smart cities are increasingly being imagined as cognitive learning environments, functioning through learning algorithms and nonconscious cognitive applications that are themselves modelled on the learning brain and are intended to become technogenetically co-constitutive of human brain processes and cognitive functioning. There are traces here of what Fitzgerald and Rose (2015) have termed the 'neurosocial city,' an urban environment characterized by practices that are animated by neuroscientific understandings of citizens. Retooled as a neurocomputational brain/ code/space, the emerging cognitive city is an urban environment designed to be more cognitively capable and to impress itself on the cerebral lives of citizens. In this sense, the brain/code/spaces of the smart city are themselves becoming learning environments where the neuroscientific diagrammatization of the brain has been mapped onto the spatial diagrammatics of the city. Education is one space in which learning algorithms are being deployed, but significantly illustrates the far wider implications of the neuroturn for smart cities as they are being transformed by actors such as IBM into brain/ code/spaces where nonconscious computing brains are embedded in the functioning of the environment and intended to weave into the cognitive experience of citizens. Of course, such processes should not be understood deterministically as if they would occur automatically. If, as Pykett (2013, p. 864) claims, the neuro knowledges are becoming part of a 'concerted attempt to re-imagine the human subject,' one identified in terms of the functioning of the brain, then the neuroscientific inspiration for cognitive classrooms and smart cities itself might be understood as changing how subjects are conceived, constituted, shaped, and managed - as subjects with interior plastic brains that can be optimized and modified through their exteriorization into neurocomputational cognitive systems. The neurotechnological dream of IBM is not simply to diagrammatize the brain, but to build brain-inspired applications that might transform current conceptions of the human subject. In doing so, it treats human cognition and computational cognition analogously, as systems of learning algorithms that can be constantly monitored, checked, re-wired, and optimized.

This initial exploration of IBM opens up a research agenda which would interrogate in more empirical depth the practices that brought these knowledges, techniques, and applications into being. With cognitive computing, the remaining sociological challenge is to trace how IBM and other actors have systemized the human brain as a complex neural network - in ways that align brain processes with computational processes - then proceduralized this knowledge in the design of applications that are intended to influence human neural morphology and cognitive functioning through the algorithmic learning environment itself. The plasticity of the socially learning brain modelled and codified in the learning algorithms of cognitive computing platforms is itself the product of practices performed by scientific and technical experts in concrete social and material 
circumstances, with a long genealogical provenance. Empirical research is required to detail how learning algorithms are designed to learn, to interrogate the models of the plasticity of the learning brain they operationalize, and to unpack the associated assumptions about managing, re-writing, and optimizing the brain on which they proceed.

In conclusion, the design of IBM's smarter classroom is just one instantiation of a contemporary set of algorithmic practices emerging from the fields of machine learning and cognitive computing and the associated imaginaries that animate their development. As a technological project based on a particular sociotechnical imaginary, IBM's Smarter Education ambitions exemplify its wider aspirations to produce smart cities that are conceived as 'naturally' cognitive environments, and where citizens themselves are conceived computationally in terms of their neurobiological malleability and amenability to algorithmic optimization. Its machine learning algorithms, neural networks algorithms, and other neurocomputational techniques of cognitive modelling are being designed to become part of urban environments in which 'an object need not be alive or conscious to function as a cognitive agent' (Hayles, 2014, p. 216). A novel kind of neurocomputational biopolitics is emerging from such practices, whereby the learning brain is imagined to be interacting with, and activated by, learning algorithms and the computing devices they enact in new kinds of brain/code/spaces. The potential consequences of such neurocomputational spaces extend beyond education to smart cities being designed by IBM to function through unsupervised machine learning processes and brain-inspired algorithms that can learn autonomously about and from the people and things that inhabit them. Such spaces are no longer hard-coded 'programmable cities,' but more 'naturally' cognitive cities with 'human qualities.' These neurotechnological applications also register the emergence of imagined 'neurofutures' based on a 'neuro-realist' set of 'brain facts' which assume that 'mental life can be understood, mapped, visualized, maintained, managed, improved, enhanced or optimized today or in the near future in these neuro-related, brain-based ways' (Williams et al., 2011, p. 136). By hybridizing neuroscientific knowledges about neuroplasticity and neural networks with new computational techniques and practices, IBM's imaginary of Smarter Education is part of a neurofuture for cities currently in-the-making, in which mental life is understood algorithmically in terms of the plasticity of neural networks and the brain's amenability to optimization.

\section{Disclosure statement}

No potential conflict of interest was reported by the author.

\section{Funding}

This research was supported with a grant from the Economic and Social Research Council [ref: ES/ L001160/1]: https://codeactsineducation.wordpress.com/

\section{Notes on contributor}

Ben Williamson is a lecturer in the School of Social Sciences at the University of Stirling, UK. His research focuses on digital data and algorithmic technologies in education, and has been published in Big Data and Society, Critical Policy Studies, and the Journal of Education Policy. [email: ben. williamson@stir.ac.uk] 


\section{References}

Artificial Brains. (2013). DARPA SyNAPSE Program. Artificial Brains: The quest to build sentient machines. Retrieved January 11, 2013, from http://www.artificialbrains.com/darpa-synapseprogram

Batty, M., Axhausen, K. W., Giannotti, F., Pozdnoukhov, A., Bazzani, A., Wachowicz, M.,... Portugali, Y. (2012). Smart cities of the future. The European Physical Journal Special Topics, 214, 481-518.

Bayne, S. (2015). Teacherbot: Interventions in automated teaching. Teaching in Higher Education, 20(4), 455-467.

Beer, D. (2013). Popular culture and new media: The politics of circulation. London: Palgrave Macmillan.

Busso, D., \& Pollack, C. (2015). No brain left behind: Consequences of neuroscience discourse for education. Learning, Media and Technology, 40(2), 168-186.

Crang, M., \& Graham, S. (2007). Sentient cities: Ambient intelligence and the politics of urban space. Information, Communication \& Society, 10(6), 789-817.

Dunn, N., Cureton, P. \& Pollastri, S. (2014). A visual history of the future. Future of Cities: working paper. London: Foresight, Government Office for Science.

Eassom, S. (2015). IBM Watson for education. IBM Insights on Business. Retrieved April 1, 2015, from http://insights-on-business.com/education/ibm-watson-for-education-sector-deakinuniversity/

Fitzgerald, D., \& Callard, F. (2015). Social science and neuroscience beyond interdisciplinarity: Experimental entanglements. Theory, Culture \& Society, 32(1), 3-32.

Fitzgerald, D., \& Rose, N. (2015). The neurosocial city. Urban Transformations. Retrieved from http://www.urbantransformations.ox.ac.uk/debate/the-neurosocial-city/

Gabrys, J. (2014). Programming environments: environmentality and citizen sensing in the smart city. Environment \& Planning D: Society \& Space, 32, 30-48.

Gillespie, T. (2014). Algorithm. Culture Digitally. Retrieved from http://culturedigitally.org/2014/ 06/algorithm-draft-digitalkeyword/

Goffey, A. (2008). Algorithm. In M. Fuller (Ed.), Software studies: A lexicon (pp. 15-20). London: MIT Press.

Hayles, N. K. (2013). How we think: Digital media and contemporary technogenesis. London: University of Chicago Press.

Hayles, N. K. (2014). Cognition everywhere: The rise of the cognitive nonconscious and the costs of consciousness. New Literary History, 45(2), 199-220.

IBM. (2013). In the future, everything will learn. Retrieved from http://www.ibm.com/ smarterplanet/us/en/ibm_predictions_for_future/ideas/

IBM. (2014). The future of learning: Enabling economic growth. Somers, NY: IBM Corporation. Retrieved from http://www-01.ibm.com/common/ssi/cgi-bin/ssialias?subtype=WH\&infotype= SA\&appname=SNDE_ED_ED_USEN\&htmlfid=EDW03005USEN\&attachment=EDW03005 USEN.PDF.

IBM. (2015a). Education for a smarter planet. Retrieved from http://www.ibm.com/smarterplanet/ us/en/education_technology/ideas/

IBM. (2015b). What is IBM Watson? Retrieved from http://www.ibm.com/smarterplanet/us/en/ ibmwatson/what-is-watson.html

IBM Research. (2011). IBM's first cognitive computing chips mimic functions of the brain. IBM Research News. Retrieved August 18, 2011, from http://ibmresearchnews.blogspot.co.uk/2011/ 08/this-cognitive-computing-chip-taught.html

IBM Research. (2013a). The classroom will learn you: Cognitive systems will provide decision support for teachers. Retrieved from www.research.ibm.com/cognitive-computing/machinelearning-applications/decision-support-education.shtml.

IBM Research. (2013b). A symbiotic cognitive experience: Human-computer collaboration at the speed of thought. Retrieved from http://researcher.ibm.com/researcher/view_group.php?id= 5417 
IBM Research. (2014). Brain Power: Scientists at IBM research unveil a brain-inspired computer and ecosystem. Retrieved from http://www.research.ibm.com/cognitive-computing/brainpower/

IBM Research. (2015). Cognitive computing. Retrieved from www.research.ibm.com/cognitivecomputing

Jasanoff, S. (2015). Future imperfect: Science, technology, and the imaginations of modernity. In S. Jasanoff \& S.-H. Kim (Eds.), Dreamscapes of modernity: Sociotechnical imaginaries and the fabrication of power (pp. 1-35). Chicago, IL: University of Chicago Press.

Kelly, J. E. III. (2015). Computing, cognition and the future of knowing: How humans and machines are forging a new age of understanding. Somers, NY: IBM Corporation. Retrieved from http://www. research.ibm.com/software/IBMResearch/multimedia/Computing_Cognition_WhitePaper.pdf

Kelly, J. E. III, \& Hamm, S. (2014). Smart machines: IBM's Watson and the era of cognitive computing. New York, NY: Columbia University Press.

Kitchin, R. (2014a). The real-time city? Big data and smart urbanism. GeoJournal, 79, 1-14.

Kitchin, R. (2014b). Thinking critically about and researching algorithms (The Programmable City working paper 5). Retrieved from http://papers.ssrn.com/sol3/papers.cfm?abstract_id=2515786

Kitchin, R., \& Dodge, M. (2011). Code/space: Software and everyday life. London: MIT Press.

Lemke, T. (2011). Biopolitics: An advanced introduction. London: New York University Press.

Linday, G. (2013). IBM'S department for education: The company that brought you smarter cities moves into schools. Next City. Retrieved from http://nextcity.org/features/view/ibmsdepartment-of-education

Mackenzie, A. (2013). Programming subjects in the regime of anticipation: software studies and subjectivity. Subjectivity, 6(4), 391-405.

Mackenzie, A. (2015a). The production of prediction: What does machine learning want? European Journal of Cultural Studies, 18(4-5), 429-445.

Mackenzie, A. (2015b). Demis Hassabis: Mindful infrastructures and re-concatenated worlds. Retrieved from http://rian39.github.io/infrastructure/2015/02/06/Demis-Hassabis-MindfulInfrastructures.html

Merolla, J., Cassidy, A. S., Sawada, J., Akopyan, F., Jackson, B. L., Imam, N., ... Modha, D. S. (2014). A million spiking-neuron integrated circuit with a scalable communication network and interface. Science, 345(6197), 668-673.

Modha, D. (2013). Systems that perceive, think and act. The Atlantic. Retrieved June 2013, from http://www.theatlantic.com/sponsored/ibm-cognitive-computing/archive/2013/06/systemsthat-perceive-think-and-act/276708/

Modha, D. (2014). Introducing a brain-inspired computer: TrueNorth's neurons to revolutionize system architecture. IBM Research. Retrieved from http://www.research.ibm.com/articles/ brain-chip.shtml

Neyland, D. (2015). On organizing algorithms. Theory, Culture \& Society, 32(1), 119-132.

Nitta, S. (2014). Cognitive learning content: A vision for how to make learning deeply engaging as well as intuitive. IBM Insights on Business. Retrieved May 14, 2014, from http://insights-onbusiness.com/education/cognitive-learning-content-a-vision-for-how-to-make-learning-deeplyengaging-as-well-as-intuitive/).

Pickersgill, M. (2013). The social life of the brain: Neuroscience in society. Current Sociology, 61(3), 322-340.

Piety, P. J., Hickey, D. T., \& Bishop, M. J. (2014, March 24-28). Educational data sciences - Framing emergent practices for analytics of learning, organizations and systems. LAK '14, Indianapolis, IN, USA. Retrieved from http://edinfoconnections.com/wp-content/uploads/2014/01/ Educational-Data-Sciences-Feb-9.pdf

Pykett, J. (2013). Neurocapitalism and the new neuros: using neuroeconomics, behavioural economics and picoeconomics for public policy. Journal of Economic Geography, 13, 845-869.

Pykett, J. (2015). Brain culture: Shaping policy through neuroscience. Bristol: Policy Press.

Rose, N. (2016). Reading the human brain: How the mind became legible. Body and Society. doi:10. $1177 / 1357034 X 15623363$

Rose, N., \& Abi-Rached, J. (2013). Neuro: The new brain sciences and the management of the mind. Oxford: Princeton University Press. 
Rose, N., \& Abi-Rached, J. (2014). Governing through the brain: Neuropolitics, neuroscience and subjectivity. The Cambridge Journal of Anthropology, 32(1), 3-23.

Service, R. (2014). The brain chip. Science, 345(6197), 614-616.

Siemens, G. (2013). Learning analytics: The emergence of a discipline. American Behavioral Scientist, 57(10), 1380-1400.

Terdiman, D. (2015). IBM using Watson to build a "Siri For Cities." Fast company. Retrieved July 22, 2015, from http://www.fastcompany.com/3048905/app-built-with-ibms-watson-is-like-aspecialized-siri-for-cities

Thrift, N. (2014). The sentient city and what it may portend. Big Data \& Society, 1(1). doi:10.1177/ 2053951714532241

Williams, S., Katz, S., \& Martin, P. (2011). The neuro-complex: Some comments and convergences. Media Tropes, 3(1), 135-146.

Williamson, B. (2015). Educating the smart city: Schooling smart citizens through computational urbanism. Big Data \& Society, 2(2), 1-13. doi:10.1177/2053951715617783 